\title{
Accurate Numerical Differencing near a Polar Singularity of a Skipped Grid
}

\author{
R. JAMES PURSER \\ Cooperative Institute for Meteorological Satellite Studies, University of Wisconsin, Madison, Wisconsin
}

(Manuscript received 4 May 1987, in final form 2 November 1987)

\begin{abstract}
For a model using spherical coordinates the presence of a polar singularity presents a number of numerical problems that deserve careful attention. Retaining all zonal Fourier components near a pole necessitates an unduly short timestep if advection or adjustment are treated explicitly. Even if both processes are treated implicitly the resolution of very small structures would require inordinately more computational effort to satisfy the implicit equations here than at other more typical portions of the grid. This difficulty is usually avoided by reducing the longitudinal angular resolution near each pole by Fourier filtering or else by deploying a "skipped grid" with progressively fewer active grid points per latitude circle at higher latitudes. While successfully easing the restriction on the timestep, these measures degrade the accuracy of the zonal gradients in the immediate vicinity of each pole unless special precautions are taken.

This paper examines the scale-dependence, averaged over orientations, of truncation error associated with conventional zonal interpolation and differencing formulae applied to the skipped grid. A remedy is proposed for the serious errors encountered and is shown to result in substantial improvements in the attainable accuracy. The method involves the smooth interpolation of zonally high frequency data to the innermost circle of latitude from the surrounding circle of data where the longitudinal resolution is higher.
\end{abstract}

\section{Introduction}

The simplest numerical grid covering the entire sphere is the familiar latitude-longitude grid, but the tight clustering of grid points and the large curvature of latitude lines near the polar singularities necessitate special measures there if such a grid is to be used for a numerical simulation of the atmosphere. In most atmospheric models the timestep is restricted by a Courant-Friedrichs-Lewy (CFL) criterion determined essentially by the shortest resolved scale divided by the largest phase speed of modes treated explicitly. Rather than allowing the atypically high resolution near the poles to dictate the timestep it is common practice to filter all but the first few Fourier components of dynamical variables near the poles between successive steps of the integration. This filtering may be accomplished either in the Fourier domain (multiplication by a frequency-dependent factor) or in the grid-point domain (convolution with a suitably smooth function). The effects of various filtering techniques on model accuracy have been compared in the studies of Umscheid and Sankar-Rao (1971) and Takacs et al. (1981). It is found that Fourier filtering is an expedient way of allowing longer time steps when the spatial differencing is of comparatively low order of accuracy (Williamson, 1976). The CFL criterion may also be relaxed by re-

Corresponding author address: Mr. R. James Purser, Met 011 Meteorological Office, London Road, Bracknell, Berkshire RG12 2SZ, United Kingdom. ducing the number of grid points per line of latitude near the poles as first suggested by Kurihara (1965) but truncation errors become unacceptably large near the poles (Sankar-Rao and Umscheid, 1969; Shuman, 1970), prompting some investigators to crowd more grid points into the polar regions than in the standard Kurihara grid (Dey, 1969; Sankar-Rao and Umscheid, 1969; Umscheid and Bannon, 1977). When high-order differencing is adopted, the reduction or contamination of available data at the first latitude circle implied by Fourier filtering or the thinning of grid points renders the usual "high order" methods ineffective. The diffculties are further compounded by the extreme curvature of the inner circle of data whose effects are discussed in Shuman (1970), Williamson and Browning (1973), Kalnay-Rivas (1976).

It appears that a high order of accuracy and a reasonably unrestrictive CFL criterion are mutually incompatible in a grid-point model, but it will be shown that there is a way out of the apparent dilemma. In the proposed approach, which will be discussed in terms of a Kurihara-type "skipped-grid" the "active" grid points, whose values are calculated from the dynamics, are still depleted as the pole is approached but at the latitude circle adjacent to the pole high frequency information is interpolated through an assumption of smoothness from the surrounding more numerous active grid points. This enables zonal (longitudinal) derivatives to be calculated with adequate precision. The method is systematic and admits several generalizations. 
The accuracy of the zonal derivatives is evaluated in terms of a simple statistic representing a normalized root-mean-square (rms) error for each total wavenumber of Fourier components in a local plane-projection of the polar region, with the mean taken over all possible orientations of the wave vector. It is found that the orientation-averaged truncation errors of the new proposed differencing method compare adequately with the corresponding statistics obtained from conventional high-order accurate difference operators applied to the straight-line data (e.g., the longitude lines of data). It is evident that the method proposed overcomes the numerical difficulties caused by a grid singularity and allows consistently accurate differencing to be achieved everywhere.

The methods of analysis applied in this paper also shed some light on the proper role of filtering in a spherical coordinate model. In particular it is noted that while a band-limited function (having no Fourier components beyond a certain critical absolute wavenumber) implies that all straight cross sections are also band limited, which justifies selective low-pass filtering of poorly resolved structures of a Cartesian grid model, it does not imply that small circular sections are devoid of high frequency components. These high frequency components are present and are necessary in a polar coordinate model in order to evaluate zonal derivatives accurately. The topic of Fourier filtering and its effects on the accuracy of zonal differencing is the subject of a companion paper (Purser, 1988).

\section{Construction of accurate differencing schemes for a skipped grid}

The sphere will be assumed marked out with lines of latitude and longitude of equal angular spacing to form the "full grid". The "active" points form a subset at which the dynamics are computed while the com- plement of "passive" points represent the points where values are obtained by interpolation from the neighboring active points. Each pole is an active point and is surrounded by an even number (at least eight) of active points occupying the line of latitude we shall refer to as "circle 1". Around this are twice as many points occupying "circle 2". High-order differencing at the poles themselves presents absolutely no problem as the lines of data radiating from them are regular and as "straight" as spherical geometry will allow. Away from the poles where the curvature of latitude circles is small, accurate differencing is also just a matter of applying the standard centered difference formula. Only in the immediate neighborhoods of the poles is the curvature of latitude circles and the paucity of data severe enough to warrant a special technique, so this section will deal specifically with the problem of extracting the zonal derivatives at circle 1 .

To provide an explicit example of the construction and analysis of interpolation and differencing methods near the polar singularity we shall examine a particular grid which happens to possess eight-fold symmetry about the pole. However, it should be emphasized that the same techniques and general conclusions apply to other skipped grid arrangements. Figure 1 shows the polar region of a skipped grid having eight active points (crosses) at circle 1. Except in section 4 we assume that effects of sphericity may be ignored so that, in effect, we are working in plane polar coordinates. For convenience take the standard "grid-unit" defined by the radial separation of successive circles to be unity and express any position vector either by polar coordinates, $(r, \theta)$ or by pole-centered Cartesian coordinates $(x, y)$ where

$$
\begin{aligned}
& x=r \cos \theta, \\
& y=r \sin \theta .
\end{aligned}
$$

Given a smooth function of position, $f(x, y)$, its Taylor series about the pole,

$$
\begin{gathered}
f(x, y)=A_{00}+\left(A_{1,0} x+A_{0,1} y\right)+\left(A_{2,0} \frac{x^{2}}{2}+A_{1,1} x y+A_{0,2} \frac{y^{2}}{2}\right)+\left(A_{3,0} \frac{x^{3}}{6}+A_{2,1} \frac{x^{2} y}{2}+A_{1,2} \frac{x y^{2}}{2}+A_{0,3} \frac{y^{3}}{6}\right) \\
+\left(A_{4,0} \frac{x^{4}}{24}+A_{3,1} \frac{x^{3} y}{6}+A_{2,2} \frac{x^{2} y^{2}}{4}+A_{1,3} \frac{x y^{3}}{6}+A_{0,4} \frac{y^{4}}{24}\right)+\mathrm{O}\left(r^{5}\right),
\end{gathered}
$$

may be expressed equivalently as the series,

$$
\begin{aligned}
f(r \cos \theta, & r \sin \theta)=a_{0,0}+r\left(a_{1,1} \cos \theta+b_{1,1} \sin \theta\right)+r^{2}\left(a_{2,0}+a_{2,2} \cos 2 \theta+b_{2,2} \sin 2 \theta\right)+r^{3}\left(a_{3,1} \cos \theta+b_{3,1} \sin \theta\right. \\
& \left.+a_{3,3} \cos 3 \theta+b_{3,3} \sin 3 \theta\right)+r^{4}\left(a_{4,0}+a_{4,2} \cos 2 \theta+b_{4,2} \sin 2 \theta+a_{4,4} \cos 4 \theta+b_{4,4} \sin 4 \theta\right)+\mathrm{O}\left(r^{5}\right) .
\end{aligned}
$$

Of the terms written out in (2.3) that contribute to the values of intermediate passive points on circle 1 or to the zonal derivative,

$$
\left.\left.\frac{\partial}{\partial y}\right|_{(x, y)=(1,0)} \equiv \frac{\partial}{\partial \theta}\right|_{(r, \theta)=(1,0)},
$$

only $r^{4} b_{4,4} \sin 4 \theta$ is undetectable from the eight active data points of circle 1 . This term, being fourth order in $r$, is responsible for the fourth order of accuracy of zonal interpolation and for the third order of accuracy of zonal derivatives obtained by the "cyclic" interpolation and differencing formulae constructed solely from the first eight resolved Fourier components. It is to improve these relatively low orders of accuracy that 


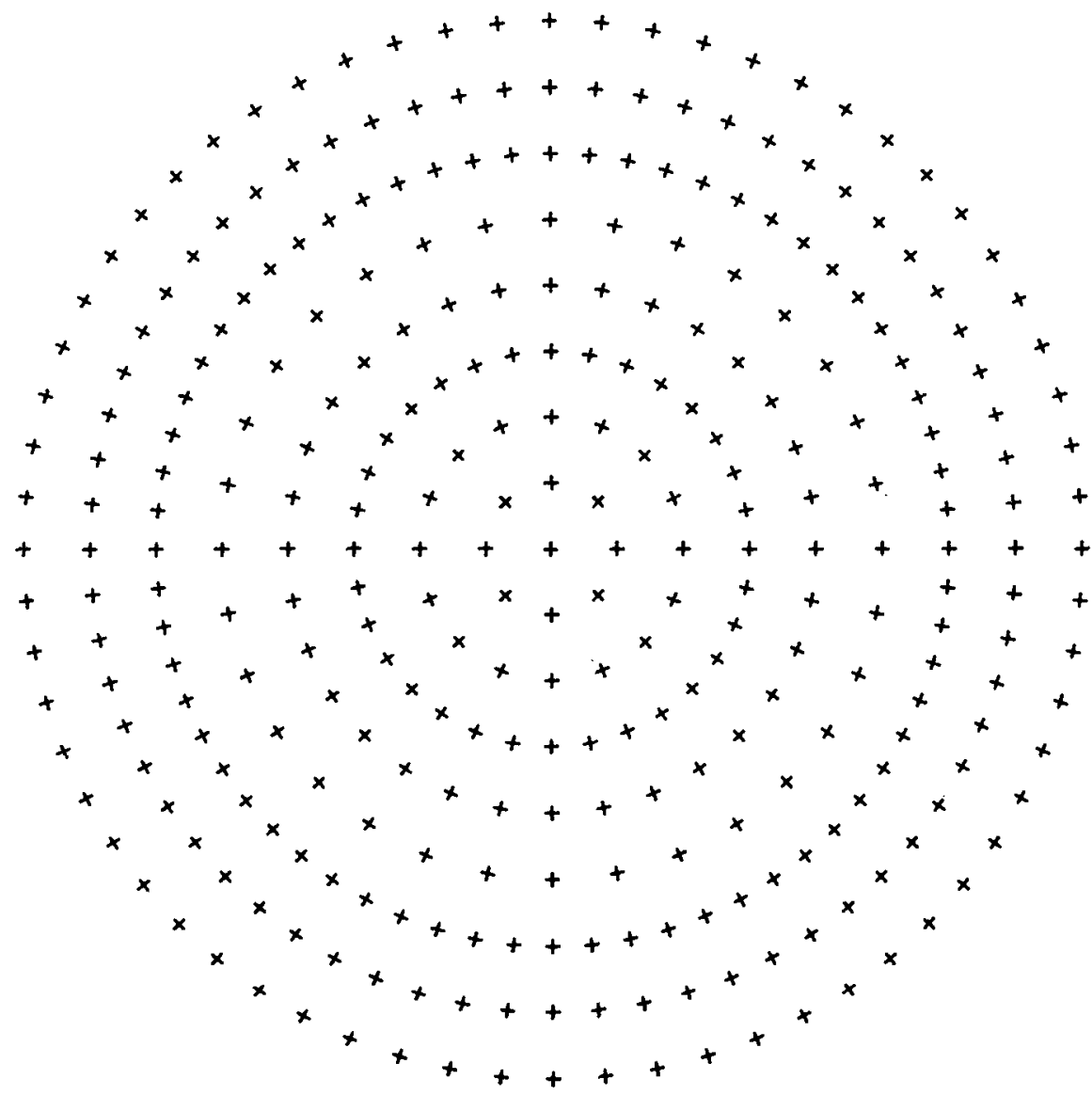

FIG. 1. Arrangement near the pole of the skipped grid with eight-fold symmetry.

we turn to the second circle of latitude for additional information to enable us to compensate for these errors.

Note that in expansions of the form (2.3) for smooth functions the earliest appearances of terms $\cos m \theta$, $\sin m \theta$ are always in association with the radial factors $a_{m, m} r^{m}, b_{m, m} r^{m}$, regardless of integer $m$. Since these terms converge to zero toward the pole less rapidly than all other terms varying as $\cos m \theta$ or $\sin m \theta$ it is fair to conclude that for smooth functions, the radial variation of the zonal Fourier coefficients $a_{m}(r), b_{m}(r)$, at wavenumber $m$ in the expansion,

$f(r \cos \theta, r \sin \theta)$

$$
=a_{0}(r)+\sum_{m=1}^{\infty}\left[a_{m}(r) \cos m \theta+b_{m}(r) \sin (m \theta)\right]
$$

are well approximated at small $r$ and sufficiently large $m$ by

$$
\left.\begin{array}{l}
a_{m}(r) \approx a_{m, m} r^{m} \\
b_{m}(r) \approx b_{m, m} r^{m}
\end{array}\right\}
$$

The assumption that (2.6) holds for the zonal Fourier components resolved by circle 2 , but not by circle 1 , justifies approximating $b_{4}(1), a_{5}(1), \ldots a_{8}(1)$ by the interpolated values:

$$
\left.\begin{array}{c}
b_{4}^{*}(1)=b_{4}(2) / 2^{4}, \\
a_{5}^{*}(1)=a_{5}(2) / 2^{5}, \\
\vdots \\
a_{8}^{*}(1)=a_{8}(2) / 2^{8} .
\end{array}\right\}
$$

For a generalization of this interpolation formula involving additional circles at latitudes see Purser (1988).

To obtain coefficients for the interpolation of additional data to circle 1 we assume that the higher zonal wavenumber structures are provided solely by coefficients $\left[b_{4}^{*}(1), a_{5}^{*}(1), b_{5}^{*}(1), \ldots A_{8}^{*}(1)\right]$, of $(2.7)$ while the lower zonal wavenumber structures are determined by the unique set of coefficients $\left[a_{0}^{*}(1), a_{1}^{*}(1), b_{1}^{*}(1) \ldots\right.$ $\left.a_{4}^{*}(1)\right]$ that ensure that the reconstructed function,

$$
\begin{aligned}
\bar{f}_{1}(\theta)=a_{0}^{*}(1)+\left(\sum_{m=1}^{7} a_{m}^{*}(1) \cos m \theta\right. & \left.+b_{m}^{*}(1) \sin m \theta\right) \\
& +a_{8}^{*}(1) \cos 8 \theta,
\end{aligned}
$$

matches the original function $f_{1}(\theta)$ at the eight active grid points, $\theta=m \pi / 4, m=0, \cdots 7$. Following a 
TABLE 1. Interpolation coefficients, $I_{1, \phi} ; I_{2, \phi} ;$ computed for the intermediate points of circle 1 in a skipped grid with eight-fold symmetry.

\begin{tabular}{lcr}
\hline \hline$\frac{8}{\pi} \phi$ & $I_{1, \phi} \times 10^{15}$ & \multicolumn{1}{c}{$I_{2, \phi} \times 10^{15}$} \\
\hline 0 & & $21,972,656,250,000$ \\
1 & $628,417,436,515,731$ & $-8,044,583,641,298$ \\
2 & & $-11,467,422,546,015$ \\
3 & $-187,075,720,333,186$ & $8,744,234,882,916$ \\
4 & & $4,394,531,250,000$ \\
5 & $83,522,329,739,912$ & $-4,196,525,654,896$ \\
6 & & $-3,181,014,953,985$ \\
7 & $-24,864,045,922,457$ & $1,543,749,413,278$ \\
8 & & $2,441,406,250,000$ \\
\hline
\end{tabular}

straight-forward manipulation of the Fourier series coefficients we may express the interpolated function at 16 evenly spaced points on circle 1 (eight active, plus eight interleaved passive points) by,

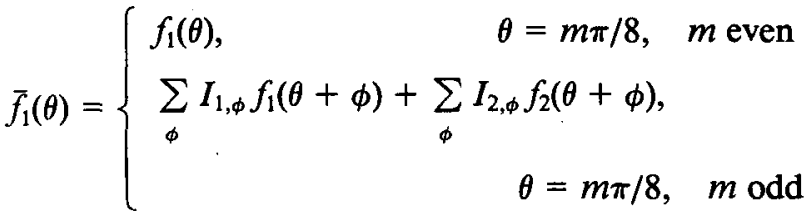

where the summations are over angles $\phi$ for which the corresponding active grid point values $f_{1}, f_{2}$ exist. We list the coefficients $I_{1, \phi}, I_{2, \phi}$ in Table 1 .

With 16 values of $f$ at our disposal on circle 1 it is now possible to use either regular high-order differencing formulae, or the 16-point cyclic differencing formula. The coefficients of the regular centered unstaggered difference schemes for equally-spaced data are given in Table 2 for even orders of accuracy up to $M=8$. The coefficients for the cyclic differencing approximation,

$$
f_{1}^{\prime}(\theta)=\sum_{\phi} G_{N, \phi} f_{1}(\theta+\phi)
$$

are

$$
\left.\begin{array}{l}
G_{N, 0}=0 \\
G_{N, \phi}=-\frac{(-)^{m}}{2} \cot \left(\frac{\phi}{2}\right), \quad \phi=\frac{m \pi}{N}, \quad m \neq 0,
\end{array}\right\}
$$

for integers $m$. These coefficients are given explicitly in Table 3 for $2 N=8$ and $2 N=16$. The following

TABLE 2. Coefficient of $(2 M)$ th-order centered unstaggered differencing on a unit grid.

\begin{tabular}{ccccc}
\hline \hline $2 M$ & $D_{M, 1}$ & $D_{M, 2}$ & $D_{M, 3}$ & $D_{M, 4}$ \\
\hline 2 & $1 / 2$ & & & \\
4 & $2 / 3$ & $-1 / 12$ & & \\
6 & $3 / 4$ & $-3 / 20$ & $1 / 60$ & \\
8 & $4 / 5$ & $-1 / 5$ & $4 / 105$ & $-1 / 280$ \\
\hline
\end{tabular}

TABLE 3. Coefficients for unstaggered cyclic differencing on circles with eight points $(N=4)$ and with 16 points $(N=8)$. Note that $G_{N, \phi}$ $=-G_{N_{1}-\phi}$.

\begin{tabular}{crr}
$\frac{8}{\pi} \phi$ & \multicolumn{1}{c}{$G_{4, \phi} \times 10^{15}$} & \multicolumn{1}{c}{$G_{8, \phi} \times 10^{15}$} \\
\hline 0 & 0 & 0 \\
1 & & $2,513,669,746,062,924$ \\
2 & $1,207,106,781,186,547$ & $-1,207,106,781,186,547$ \\
3 & & $748,302,881,332,744$ \\
4 & $-500,000,000,000,000$ & $-500,000,000,000,000$ \\
5 & & $334,089,318,959,650$ \\
6 & $207,106,781,186,547$ & $-207,106,781,186,547$ \\
7 & 0 & $99,456,183,689,829$ \\
8 & 0 & 0 \\
\hline
\end{tabular}

section analyzes quantitatively the truncation errors associated with the techniques of zonal differencing and interpolation that we have discussed.

\section{Truncation error}

For differencing and interpolation schemes in one dimension it is informative to display the relative errors as a function of the wavenumber of individual Fourier components,

$$
f_{\nu}(x) \propto e^{i \nu x},
$$

of the data. The order of accuracy is then reflected in the principal exponent of the Taylor series of this error about wavenumber zero. The standard normalized errors,

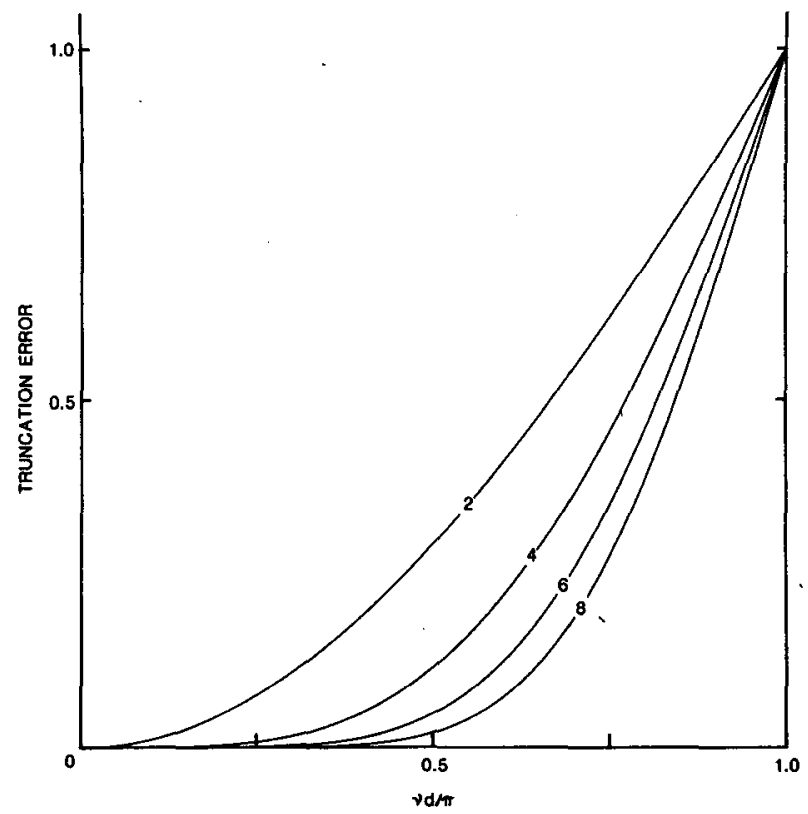

Fig. 2. Normalized truncation error as a function of wavenumber $\nu$ for centered midpoint interpolation from a uniform one-dimensional grid of spacing $d$. Graphs depict errors corresponding to schemes of even orders 2 through 8 as indicated. 


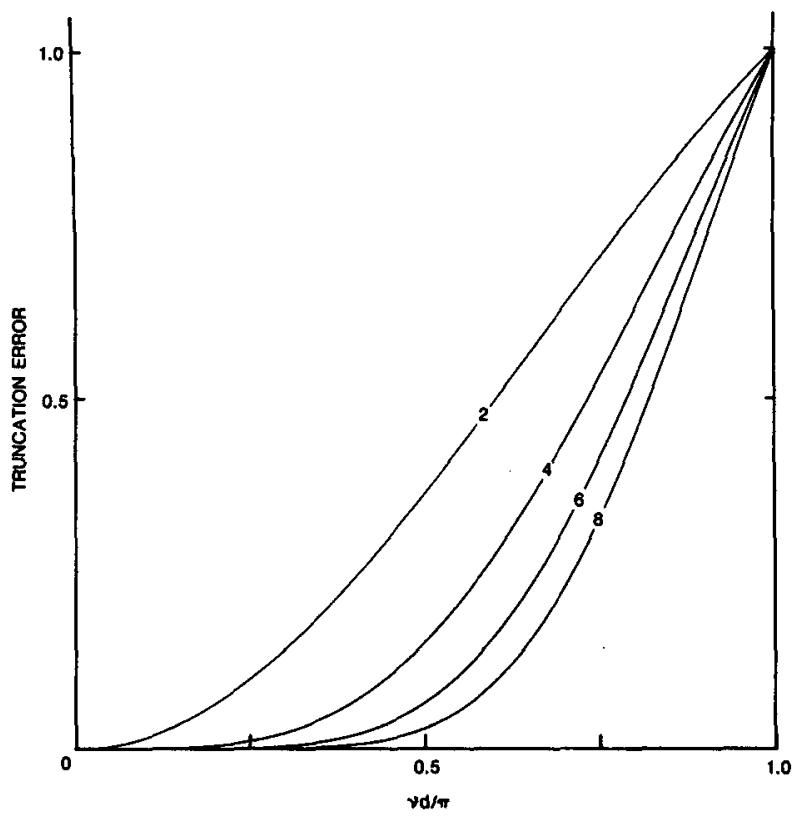

FIG. 3. Normalized truncation error as a function of wavenumber $\nu$ for centered unstaggered differencing from a uniform one-dimensional grid of spacing $d$, schemes of even orders 2 through 8 as indicated.

$$
U(\nu)=\left|\hat{D} f_{\nu}-D D f_{\nu}\right| /\left|D f_{\nu}\right|
$$

where linear functional $D$ and its discrete approximation $\hat{D}$ represent midpoint interpolation and spatial differentiation, are displayed respectively in Figs. 2 and 3. However, for the two-dimensional polar grid we have been examining it is misleading to pay any attention to the relative errors as a function of zonal wavenumbers near the pole for, as we have already seen, the higher wavenumber components diminish rapidly in amplitude towards the pole provided, as we shall always assume, the function $f(x, y)$ is smooth. A superior but more cumbersome analysis of truncation error would be obtained by observing the relative error as a function of the two wave vector components $(k, l)$, conjugate to $(x, y)$, of the Fourier components obtained from a projection of the polar region to a Cartesian tangent plane. An example of such an analysis is provided in Fig. 4 which shows contours of the error $U(k, l)$ generalizing (3.2) for zonal differencing on circle 1 by centered second-order (a); fourth-order (b); and eight point cyclic (c) formulae. It is much more convenient, though slightly less informative, to express the error as a mean squared value obtained by averaging the squared error over all orientations of the wave-vectors having the same absolute magnitude, $\nu=|\mathbf{k}|$. The averaging can be taken care of analytically, making the actual computations straight-forward and economical, regardless of the irregularities of the data-grid. The technique has been successfully applied in a semidiscrete case to the analysis of truncation errors caused by zonal Fourier filtering at high latitudes in the companion paper (Purser, 1988). Here we follow the same methods and notation and extend the analysis to a fully discrete grid. The orientation-averaged squared error $R(\nu)$ at absolute wavenumber $\nu$ incurred in the numerical evaluation $\mathcal{D}$ of a linear functional $\mathcal{D}$ was shown in section 2 of the companion paper to be equivalent to a variance,

$$
R(\nu)=\left\langle\left(D f_{\nu}-\mathscr{D} f_{\nu}\right)^{2}\right\rangle,
$$

where $f_{\nu}$ is a realization of a two-dimensional Gaussian stochastic process with autocovariance taking the form of the Bessel function of the first kind:

$$
c(\mathbf{x})=J_{0}(\mathbf{x} \nu) \equiv J_{0}(|\mathbf{x}| \nu) \text {. }
$$

When the functional $D$ is the fully discrete operator

$$
\hat{D} f \equiv \sum_{a=1}^{M} D_{a} f\left(\mathbf{x}_{a}\right)
$$

then $R(\nu)$ takes the form,

$$
R(\nu)=\sum_{\substack{a=0 \\ b=0}}^{M} D_{a} C_{\nu}^{a b} D_{b},
$$
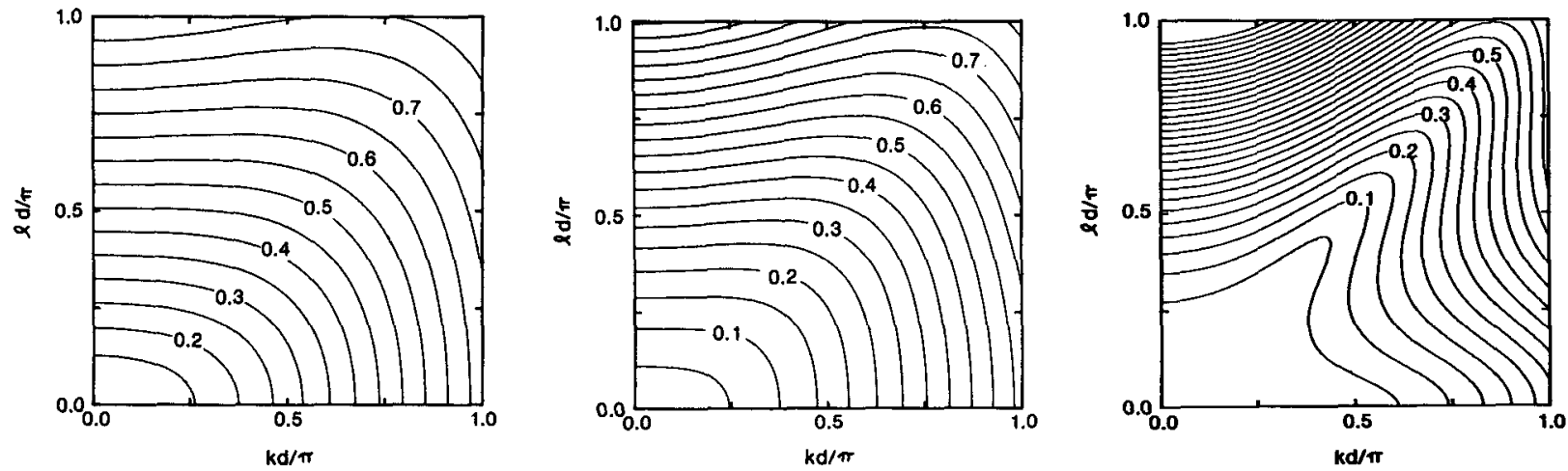

FIG. 4. Normalized absolute truncation error $U$ of zonal unstaggered differencing on circle 1 for Fourier components of the form $\exp (i[k x$ $+l y])$ contoured as a function of wave vector $(k, l)$ : (a) centered second-order; (b) centered fourth-order; (c) cyclic differencing on eight points. 
where $D_{0}=-1$ and $C_{\nu}{ }^{a b}$ are the covariances:

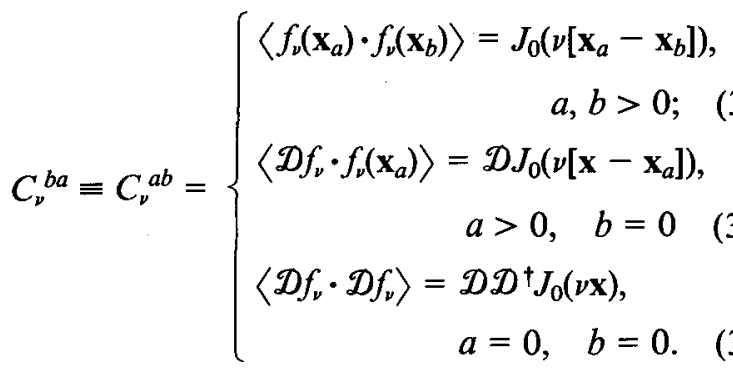

$D_{D^{\dagger}}$ is the symmetric functional obtained from $D$ by the defining property that for any function $f(\mathbf{x})$,

where

$$
\left.\begin{array}{rl}
\mathscr{D D}^{\dagger} f(\mathbf{x}) & =\mathscr{D} g(\mathbf{x}), \\
g\left(-\mathbf{x}^{\prime}\right) & =\mathscr{D}_{h_{\mathbf{x}}(\mathbf{x}),} \\
h_{\mathbf{x}}(\mathbf{x}) & =f\left(\mathbf{x}-\mathbf{x}^{\prime}\right) .
\end{array}\right\}
$$

The case of interpolation is the easiest to deal with. Suppose we are testing the accuracy of interpolation to the point $\mathbf{x}_{0}$, i.e., $\mathscr{D} f(\mathbf{x}) \equiv f\left(\mathbf{x}_{0}\right), \mathcal{D D}^{\dagger} f(\mathbf{x})=f\left(\mathbf{x}_{0}\right)$. Then (3.7a) defines $C_{\nu}{ }^{a b}$ for all $a$ and $b$. When the functional $D$ represents differentiation, say $\frac{\partial}{\partial y}$ at $\mathbf{x}=\mathbf{x}_{0}$, then

$$
\begin{aligned}
C_{\nu}{ }^{a 0} & =\left.\frac{\partial}{\partial y} J_{0}\left(\nu\left[\mathbf{x}-\mathbf{x}_{a}\right]\right)\right|_{\mathbf{x}=\mathbf{x}_{0}} \\
& =\nu J_{1}\left(\nu\left|\mathbf{x}_{0}-\mathbf{x}_{a}\right|\right) \frac{y_{a}-y_{0}}{\left|\mathbf{x}_{0}-\mathbf{x}_{a}\right|}, \\
C_{\nu}{ }^{00} & =-\left.\frac{\partial^{2}}{\partial y^{2}} J_{0}(\nu \mathbf{x})\right|_{\mathbf{x}=0}=\frac{\nu^{2}}{2} .
\end{aligned}
$$

The natural range of $\nu$ to investigate when the radial separation of grid-circles is unity is

$$
|\nu|<\pi \text {. }
$$

Larger values of $\nu$ are subject to aliasing errors. As in the companion paper, we normalize the mean-squared truncation errors by the corresponding mean-squared actual value of the functional being approximated, and take the square root of the result as the final measure:

$$
T(\nu)=\left\{R(\nu) / C_{\nu}^{00}\right\}^{1 / 2} .
$$

Before we apply this technique of analysis to the polar singularity example of section 2 , we compare (3.12) with the analogous formula (3.2) for the midpoint interpolation and unstaggered partial'differentiation for uniformly spaced collinear data. The partial derivatives are directed along the line of data, so the orientationaveraged truncation errors $T(\nu)$ for interpolation (Fig. 5) and differencing (Fig. 6) are to be compared with the corresponding one-dimensional results of Fig. 2 and Fig. 3, respectively. As before, we have tested schemes of accuracy up to eighth order. The smaller magnitude of error at a given $\nu$ in the case of $T(\nu)$ is a

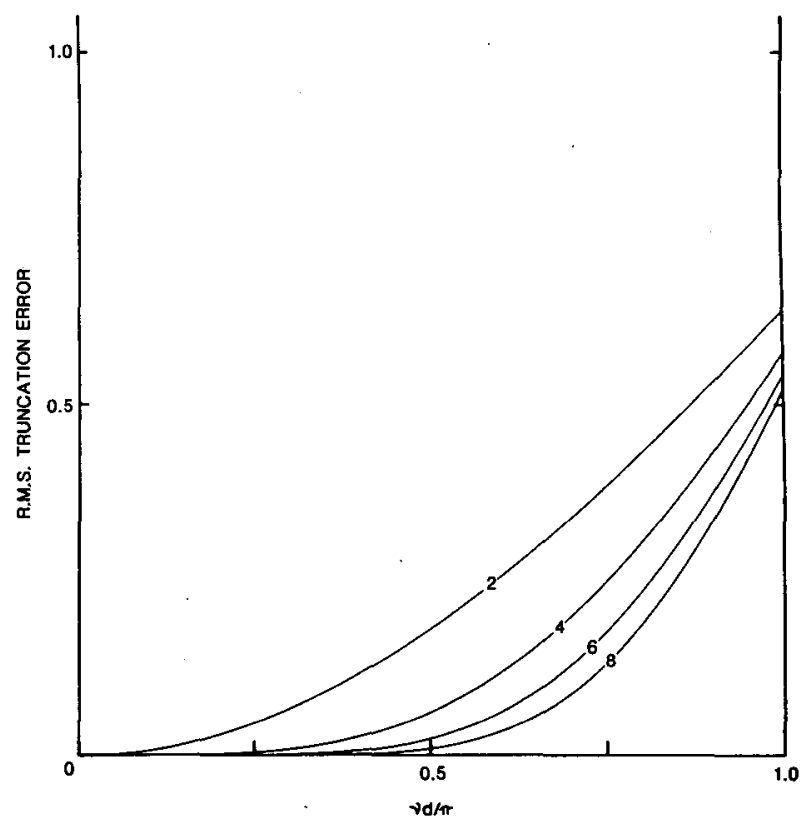

FIG. 5. Normalized orientation-averaged truncation errors of the interpolation schemes corresponding to Fig. 2 but applied to collinear data in a plane.

result of averaging over all orientations, as the Fourier components at oblique angles to the line of data cannot be distinguished from longer waves oriented parallel to the line. With this provision accepted, the comparisons among the orders of accuracy are qualitatively similar in using either $T(\nu)$ or $U(\nu)$ as the criterion. The salient characteristics of both measures of error are that the application of high order formulae in both interpolation and differencing has little impact on the errors near the Nyquist limit, $\nu=\pi$, but is extremely effective at reducing these errors in the half range $|\nu|<\pi / 2$ (corresponding to waves longer than four grid-units) which might be termed the "effective" range of resolution. It is with errors at these intrinsic scales that we shall be concerned as we return now to our main objective: assessing truncation errors of the skipped grid for various interpolation and differencing methods near the polar singularity.

The upper curves of Fig. 7 shows the orientationaveraged truncation errors $T(\nu)$ for four methods of interpolating zonally to a "skipped point" intermediate between two of the eight active grid points of the subpolar latitude circle. The three upper curves marked " 2 ", " 4 " and " $C 8$ " use only the eight data of this circle and are second order, fourth order and cyclic interpolation respectively. Compared with the straight line interpolation formulae of Fig. 5 we observe that the accuracy of interpolation is improved over most of the effective range of resolution (and is clearly better near the Nyquist limit $\nu=\pi$ ). However, for long waves, which we expect to be handled accurately, the accuracy of the second- and fourth-order schemes is degraded 


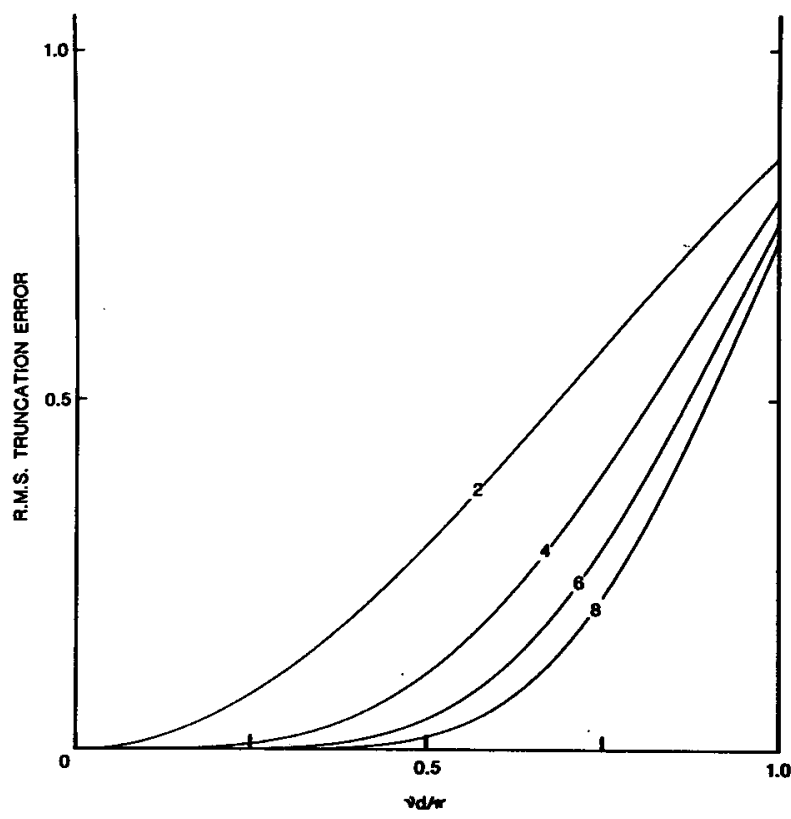

FIG. 6. Normalized orientation-averaged truncation errors of the differencing schemes corresponding to Fig. 3 but applied to collinear data in a plane.

by the curvature of the grid. Even the cyclic formula (C8) has a formal order of accuracy of only four, although it appears to be better than the straight sixth-

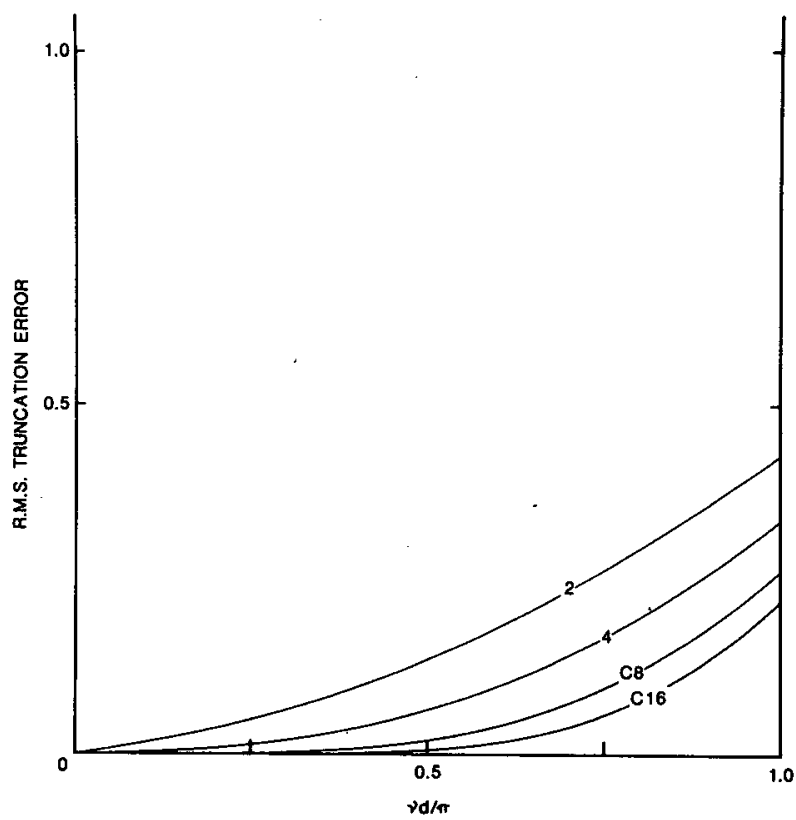

FIG. 7. Normalized orientation-averaged truncation errors of the interpolation to the eight intermediate passive points of circle 1 for a skipped grid with eight-fold symmetry. Curves 2 and 4 are for the ordinary centered midpoint interpolation from the neighboring active points. Curve $\mathrm{C} 8$ is for cyclic interpolation from the available eight active points of circle 1 . Curve $\mathrm{Cl} 6$ is the method of smooth interpolation described in section 2 using data from circle 1 and circle 2 . order method of Fig. 5 for most of the effective range. The improvement at larger wavenumbers $\nu$ is clearly due to the closer-than-unit spacing of the eight grid points. The fourth curve of Fig 7, marked (C16), shows the error associated with the interpolation (2.9) involving two circles of latitude, and shows that excellent accuracy (though formally only of order six) is attained in the range, $|\nu|<\pi / 2$.

The serious deficiencies of simple differencing near the singularity are most apparent when we consider the plots of truncation error $T(\nu)$ shown in Fig. 8 for zonal differencing approximations of orders two and four and the eight-point cyclic differencing formula (C8), all of which use only the eight active data points on circle 1 . These graphs compactly summarize the essential information provided in more complete form in the contour plots of Fig. 4. In order to judge the extent to which the curvature of the grid degrades the accuracy of differencing on circle 1, the graphs of Fig. 8 for zonal differencing should be compared with those of Fig. 6 for straight, uniformly spaced data. It is particularly disturbing that the (nominally!) second- and fourth-order schemes are not even close to being "consistent" at $\nu=0$, and the modest errors near $\nu=\pi$ are no compensation for the significant errors at large scales. The cyclic differencing formula is formally of order three in accuracy and has errors at $\nu=\pi / 2$ that would not be considered acceptable if the differencing elsewhere were of sixth or eighth order.

To augment the accuracy of zonal differencing at circle 1 we now use the additional intermediate values

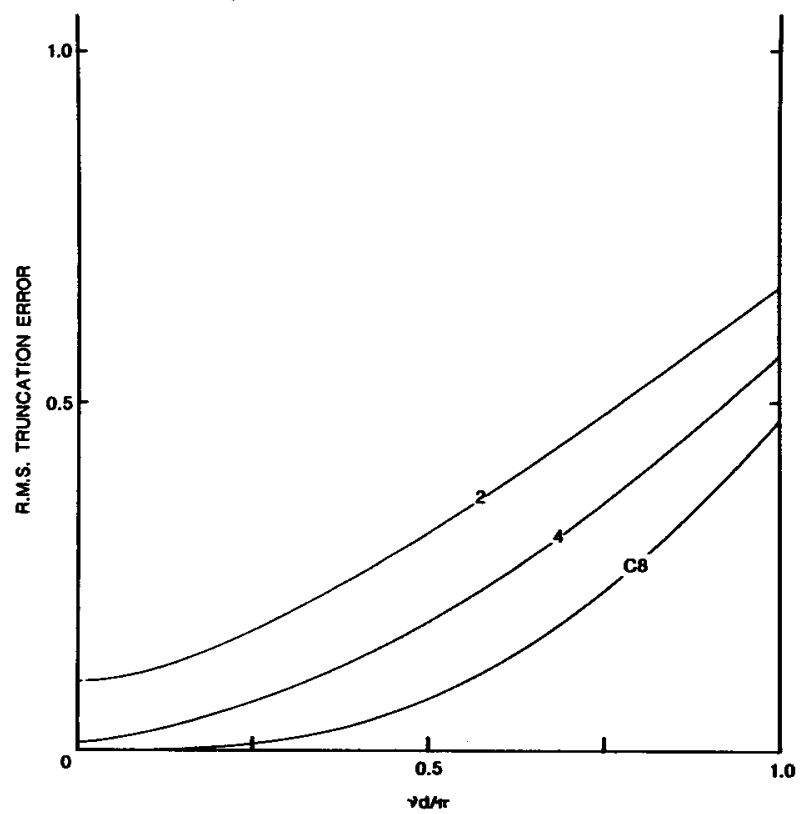

FIG. 8. Normalized orientation-averaged truncation errors of zonal differencing at circle 1 using only the eight active points of circle 1 . Curves $\mathbf{2}$ and $\mathbf{4}$ are for the ordinary unstaggered differencing formulae while $\mathrm{C} 8$ is for the eight-point cyclic formula. 
obtained by interpolation from both circle 1 and circle 2 according to the method defined by (2.9). Figure 9 shows the graphs of $T(\nu)$ for conventional centered differencing of nominal orders $2,4,6$ and 8 applied to the interpolated values $\bar{f}_{1}(\theta)$ of $(2.9)$. Also shown is the error corresponding to the application of the cyclic differencing scheme of 16 points (marked C16) to $\bar{f}_{1}(\theta)$. It is evident that, apart from the nominally secondorder scheme, which remains stubbornly inaccurate near $\nu=0$, the accuracy of differencing is now significantly improved. The closeness of the more accurate error-curves in Fig. 9 is a reflection of the fact that the gain in accuracy stems mainly from the removal of aliasing error by the interpolation process. The truncation errors for all schemes except that of second order are now of acceptable magnitudes compared with even the sixth- and eighth-order schemes for straightline data depicted in Fig. 6, although even the cyclic scheme, $\mathrm{Cl6}$, is formally only fifth-order accurate. Should it be necessary for any reason to reduce the truncation errors still further in the effective range of resolution $|\nu|<\pi /$ 2 , one could adapt the generalized interpolation formulae discussed in the companion paper to use more than just two circles of data. It would not be effective merely to increase the number of zonal Fourier components interpolated from circle 2 as the dominant

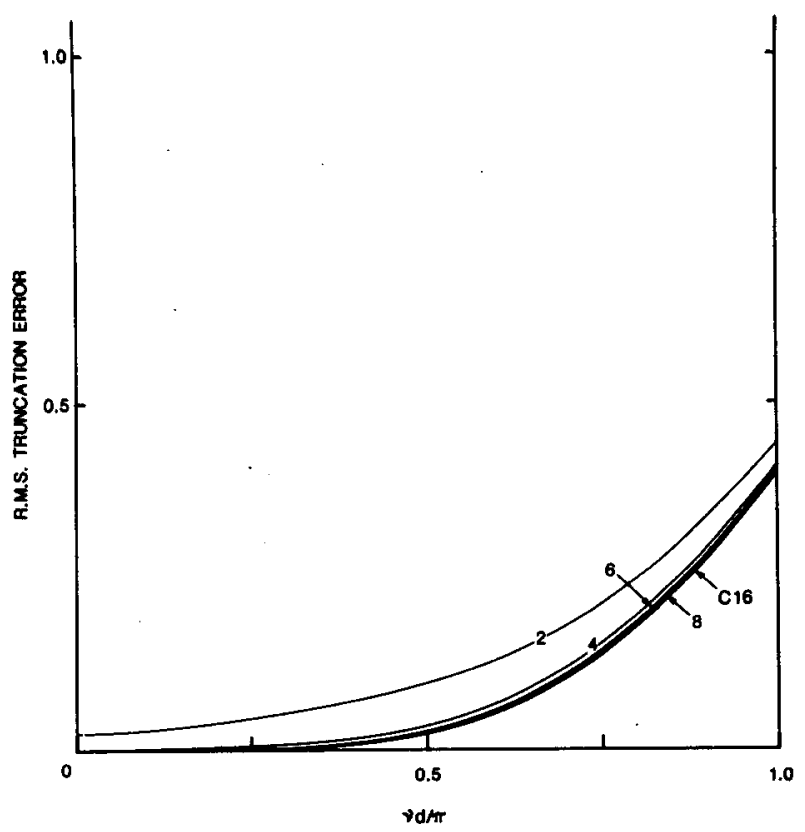

FIG. 9. Normalized orientation-averaged truncation errors of the zonal differencing on a skipped grid with eight-fold symmetry for schemes using the 16 available points of circle 1 obtained after applying the smooth interpolation method from the active points of circle 1 and circle 2 . Curves 2 through 8 are for ordinary centered unstaggered differencing, while $\mathrm{C} 16$ is for cyclic differencing from the 16 points. term in the series (2.3) not represented by the interpolation scheme $(2.8)$ is

$$
b_{6,6} r^{2} \sin 6 \theta \text {, }
$$

whose zonal wavenumber is already well resolved.

\section{Vector fields and sphericity}

The application of methods of numerical interpolation and differentiation to vector fields should take account of the rapidly changing orientations the standard vector bases in the neighborhood of the polar singularity and, in the case of zonal differentiation, the small effect due to the earth's sphericity. Let $u$ and $v$ be respectively the eastward and northward components of a vector field which we conveniently denote by the single complex quantity,

$$
w=u+i v .
$$

To "uncurl" the variation of vector-bases in the vicinity of the pole it is sufficient to multiply $w$ by the longitudedependent factor $e^{i \theta}$ :

$$
w^{\prime}(\theta)=e^{i \theta} w(\theta)
$$

The interpolation formula (2.9) can then be applied to the transformed components $w^{\prime}$ without encountering numerical problems due to the singularity in the orientation of bases. The consistent vector version of (2.9) applied to untransformed complex representations $w$ of the vector field is therefore of the same form as (2.9) but with $I_{r, \phi}$ replaced by

$$
I_{r, \phi}^{\prime}=I_{r, \phi} e^{i \phi}, \quad r=1,2 .
$$

The physically meaningful vector counterpart of the zonal component of the gradient of a scalar field is the zonal component of the covariant derivative of a vector field. Applying the zonal differencing formula to transformed data $w^{\prime}$, followed by the inverse transformation, is only correct for plane-polar coordinates but is not quite correct on the sphere because the rotation factor of (4.2) overcompensates for the relative rotation of the original bases with respect to change in longitude. The intrinsic rotation rate of "north" per unit angle of longitude at colatitude $\lambda$ is $\cos \lambda$, not unity, as can be verified by considering the geometry of the tangentcone for colatitude $\lambda$ and the intrinsic curvature of that latitude circle (Shuman, 1970). With proper allowance made for sphericity, the vector version of the differencing formula (2.10) for the covariant derivative (with respect to longitude $\theta$ ) of a vector field $w$ at colatitude $\lambda$ is therefore:

$$
\partial_{\theta} w_{\lambda}(\theta)=\sum_{\phi} G_{N, \phi}^{\prime} w_{\lambda}(\theta+\phi)-i(1-\cos \lambda) w_{\lambda}(\theta) \text {, }
$$

where

$$
G_{N, \phi}^{\prime}=G_{N, \phi} e^{i \phi}
$$


Although the added correction term in (4.4) is normally small, it should be retained nevertheless in order to guarantee consistency.

\section{Discussion}

The truncation errors incurred by performing zonal interpolation and differencing on a latitude-longitude skipped grid are examined in terms of the orientationaveraged squared-error. The results of this analysis concerning the degradation of accuracy of nominally low-order differencing methods are consistent with the previous finding of Shuman (1970) and Williamson and Browning (1973) that show that significant errors occur for zonal differencing near the pole when the separation in longitude of the data points is relatively large. It is also shown that to correct the problem it is not enough to use all the data from the first circle (i.e., eight values in the particular example chosen for study) in the "cyclic" differencing formula, which is exact for all the zonal Fourier terms resolved. However, augmenting the limited information at circle 1 by a smooth interpolation of the higher frequencies resolved at circle 2 does bring about a substantial improvement in the accuracy, both of the interpolated function values at the intermediate "passive" grid points of circle 1 (i.e., values that are necessary for the meridional differencing further out), and more especially, in the zonal differencing at circle 1 . Because we are not increasing the resolution of the grid by this method we are not violating any CFL-type stability conditions unless they were already violated without this refinement. (In the related context of high latitude filtering the corresponding assertion was verified by a simple numerical experiment presented in Purser (1988).

The main competition the global grid-point forecasting models must face are'the spectral methods (Bourke, 1972) which, at significantly less than the theoretical resolution of conventional grid-point models, have been found to perform as well, or better (Girard and Jarraud, 1982). This suggests that the principal outstanding numerical deficiencies of grid-point models are not related merely to grid-scale effects but also to their treatment of the scales they resolve well. The relatively low order of differencing (second or fourth) typical of operational grid point models is known to give rise to significant truncation errors at scales well resolved by the grid and, as we have seen, these errors become severe near the poles whenever the number of zonal degrees of freedom is restricted to accommodate the CFL criterion. In this paper, emphasis has deliberately been given to the significance of truncation errors at these larger scales (i.e., wave lengths exceeding four grid units) in order to draw attention to the potential for improving the accuracy at these scales by a combination of high-order differencing (sixth or eighth) on the grid as a whole, together with a special procedure to take care of the polar problem. Conservation principles have not been addressed explicitly because it is felt that, at the scales of importance the conservation laws that follow from the governing equations (or suitable idealizations of them) will necessarily be satisfied to a sufficient degree of accuracy when numerical representations of the governing equations themselves are accurate. However, the cumulative effects of even a weak violation of a conservation law may be serious over the course of an extended integration, as was shown by Takacs and Balgovind (1983) in their study of various methods of high-latitude filtering. Further work is needed, probably with a global model, to assess the significance of minor but persistent violations of conservation laws in a model whose spatial differencing is consistently high-order.

Staggered differences at a given order of accuracy are uniformly more accurate than unstaggered differences and especially so at the grid scale. This fact is customarily exploited to treat some of the processes (e.g., the effect of divergence on the mass tendency) with relatively high precision; however, it is inevitably at the expense of accuracy elsewhere (e.g., geostrophic balance) since a "natural" staggering for one group of terms in the dynamical equations implies the need for horizontal "midpoint" averaging operators elsewhere which have their own severe problems for grid-scale components, so overall the gains in accuracy achieved by staggering the variables of a complete model are rather less impressive when compared against the superiority of staggered over unstaggered differencing on a line. Because of this and the complexity inherent in the consistent implementation of high-order differencing in more than one dimension when variables are spatially staggered (Kalnay-Rivas, 1978) no attempt has been made to examine the errors resulting from any arrangement of grid points more complicated than that shown in Fig. 1. The only obstacle to achieving consistently accurate horizontal differencing in this grid is removed by adopting the special interpolation procedure of section 2, or its generalization noted in Purser (1988), at the subpolar latitude circles. It remains for future work to decide whether the methods advocated here enable grid-point forecasting models to match the quality of their spectral competition at the scales properly resolved.

Acknowledgments. The author is grateful to Ms. JanWaite-Schuster for typing the manuscript and to Mr. Anthony Wendricks for assistance with the preparation of figures. This research was supported by NOAA Grant NA84AA-H-00028.

\section{REFERENCES}

Bourke, W., 1972: An efficient, one-level, primitive-equation spectral model. Mon. Wea. Rev., 100, 683-689. 
Dey, C. H., 1969: A note on global forecasting with the Kurihara grid. Mon. Wea. Rev., 97, 597-601.

Girard, C., and M. Jarraud, 1982: Short and medium range forecast differences between a spectral and grid point model. An extensive quasi-operational comparison. Tech. Rep. 32, ECMWF, Reading RG2 9AX, England, $178 \mathrm{pp}$.

Kalnay-Rivas, E., 1976: High-latitude truncation errors of box-type primitive equation models. Mon. Wea. Rev., 104, 1066-1069.

- 1978: Comments on "U.S. Navy Fleet Numerical Weather Central operational five-level fourth-order primitive equation model." Mon. Wea. Rev., 106, 1021-1022.

Kurihara, Y., 1965: Numerical integration of the primitive equations on a spherical grid. Mon. Wea. Rev., 93, 399-415.

Purser, R. J., 1988: Degradation of numerical differencing caused by Fourier filtering at high latitudes. Mon. Wea. Rev., 116, 10571066.

Sankar-Rao, M., and L. Umscheid, 1969: Tests of the effect of grid resolution in a global prediction model. Mon. Wea. Rev., 97, 659-663.
Shuman, F. G., 1970: On certain truncation errors associated with spherical coordinates. J. Appl. Meteor., 9, 564-570.

Takacs, L. L., and R. C. Balgovind, 1983: High-latitude filtering in global grid-point models. Mon. Wea. Rev., 111, 2005-2015.

__ _ M. Iredell and E. Kalnay, 1981: On the effect of high latitude filtering in global grid-point models. Preprints, Fifth Conference on Numerical Weather Prediction, Monterey, Amer. Meteor. Soc., 116-121.

Umscheid, L., and M. Sankar-Rao, 1971: Further tests of a grid system for global numerical prediction. Mon. Wea. Rev., 99, 686-690.

- , and P. R. Bannon, 1977: A comparison of three global grids used in numerical prediction models. Mon. Wea. Rev., 105, 618635.

Williamson, D. L., 1976: Linear stability of finite-difference approximations on a uniform latitude-longitude grid with Fourier filtering. Mon. Wea. Rev., 104, 31-41.

- and G. L. Browning, 1973: Comparison of grids and difference approximations for numerical weather prediction over a sphere. J. Appl. Meteor., 12, 264-274. 